Cyberpsychology, Behavior, and Social Networking

Volume 00, Number 00, 2020

(c) Mary Ann Liebert, Inc.

DOI: 10.1089/cyber.2019.0445

\title{
Gaming and Gaming Disorder: A Mediation Model Gender, Salience, Age of Gaming Onset, and Time Spent Gaming
}

\author{
Frank D. Buono, PhD, Erina Paul, PhD, ${ }^{2}$ Matthew E. Sprong, PhD, ${ }^{3,4}$ Emma C. Smith, BA, ${ }^{1}$ \\ Amir Garakani, MD, and Mark D. Griffiths, $\mathrm{PhD}^{5}$
}

\begin{abstract}
Females in empirically based peer-reviewed studies of internet gaming disorder are underrepresented, despite evidence that there are only minor gender disparities present in online gaming. Moreover, few studies have specifically evaluated adult gender effects, within a formal diagnosis of internet gaming disorder, and behavioral motivation, as defined by the reinforcing behavioral function. A mediation analysis evaluated the relationship between gender, behavioral motivation, and the diagnostic features in online gaming among adults to understand the impact of motivation on videogame playing. The present study interviewed 304 adults (aged over 18 years) in which 178 identified as female. Participants completed the Video Game Functional AssessmentRevised (VGFA-R) and the 20-item Internet Gaming Disorder Test (IGDT-20) via an online survey. Results showed that number of hours played per week, and sub-factors of the VGFA-R differed between gender, indicating that the function and the maintaining of videogame play is essential in evaluating videogame addiction. These findings support and extend the literature's limited findings concerning gender and online gaming.

Keywords: Gender, Internet Gaming Disorder, Motivation, Diagnosis, Mediation Analysis
\end{abstract}




\section{Introduction}

The prevalence of internet gaming disorder (IGD) is widely variable across adults ${ }^{1,2}$ with prevalence rates at around $1 \%-5 \%{ }^{3}$. Females are relatively underrepresented in empirically-based peer-reviewed studies ${ }^{4,5}$, despite evidence that there are only minor gender disparities found in online gaming. Recent statistics reported by the Entertainment Software Association ${ }^{6}$ indicated that up to $45 \%$ online gamers are female, increasing $12 \%$ since 2008 . Additionally, females are more likely to report poorer mental health issues and higher psychological distress than males ${ }^{7,8}$, along with potential difference in the preoccupation with gaming and associated activities, and the constant thinking about gaming (even when not actually playing) ${ }^{9}$. Furthermore, there are differences across gender in relation to motivation ${ }^{10,11}$ (i.e., a primary motivation for females is to maintain relationships or to engage in the social perspective of game play ${ }^{12-14}$, as opposed to males who engage predominantly for achievement-based reasons).

Understanding the reinforcing behavioral motivations by gender within online gaming is underrepresented within the field. There are several standardized assessments that integrate IGD criteria, based on the fifth edition of the Diagnostic and Statistical Manual of Mental Disorders $(\mathrm{DSM}-5)^{11,15}$, yet there is little research evaluating the behavioral perspective of online gaming motivation across gender when evaluating duration of active engagement via assessment tools such as the IGDT-20 ${ }^{16}$. By conducting a mediation analysis will allow for interpretation of how behavioral motivation can affect the criterion of IGD and the differences of gender in videogame play $^{17}$. Therefore, the present study examined the impact of behavioral motivation utilizing the Video Game Functional Assessment-Revised (VGFA-R ${ }^{18}$ ) in relation to duration of time impact of hours of play, gender, and the risk of IGD utilizing the IGDT-20. Based on previous research ${ }^{5,19}$ 
it was hypothesized that females would have higher levels of attention-maintained behavioral motivation, while males would have higher escape-maintained behavioral motivation.

\section{Methods}

\section{Participants}

A total of 320 participants met inclusion criteria (see Sprong et al. ${ }^{19}$ ) and volunteered to participate in the study; 304 remained after data cleaning. The average age of the total participants that completed the study was 29.8 years $(S D=9.82)$, and 178 identified as female $(56 \%)$. The remaining demographic information is shown in Table 1.

\section{Materials}

Participants completed the Video Game Functional Assessment-Revised (VGFA-R) on a 24-item Likert scale that assesses four functions that sustain videogame play: (i) social attention, (ii) tangible/intangible rewards, (iii) escape/avoidance of demands or pain, and sensory stimulation (Buono et al., 2016) ${ }^{20}$. The assessment comprised one of seven responses indicating degrees of agreement or disagreement to the statement $(1=$ Never, $2=$ Almost never, $3=$ Seldom, $4=$ Half of the time, 5=Usually, $6=$ Almost always, $7=$ Always). The assessment included six questions relating to each of the four behavioral functions for a total of 24 questions. The VGFA-R had excellent overall reliability $(\alpha=.927)$ and individual reliabilities for each functions: attention $(\alpha=.91)$, escape $(\alpha .80)$, tangible $(\alpha=.84)$, sensory $(\alpha=.76)^{18}$.

The 20-item Internet Gaming Disorder Test (IGDT-20) assesses six components of addictive behavior (i.e., salience, mood modification, tolerance, withdrawal symptoms, conflict, and relapse) to which risk of IGD can be made according to the DSM-5 IGD criteria ${ }^{15}$. The IGDT20 includes three to five questions that relate to each of the six components of addictive behavior: salience $(n=3)$, mood modification $(n=3)$, tolerance $(n=3)$, withdrawal symptoms $(n=3)$, conflict 
$(n=5)$, and relapse $(n=3)$. Participants choose one of five responses on a Likert scale $(1=$ strongly agree, $2=$ agree, $3=$ neither agree nor disagree, 4=disagree, 5=strongly disagree) and the overall internal consistency was excellent in the present study $(\alpha=.93)$.

\section{Procedure}

The present study was approved by the research team's institutional review board before participant recruitment. All participants received a recruitment email that provided information about the study including its purpose, the length of participation in the study, the inclusion criteria, (e.g., being aged 18 years + ), and information about study discontinuation and reimbursement for participation. The email included the recruitment script and the individuals interested in participating in the study were instructed to click a link at the end of the recruitment script that directed them to the study materials via the Qualtrics software program. All participants were debriefed after completion of the assessments and were entered in a prize draw for five \$50(US) Amazon gift-cards.

\section{Data analysis}

Mediation analyses were performed on how covariates influenced the relationship between the predictor and outcome variables. The Average Causal Mediation Effects (ACMEs) and Average Direct Effects (ADEs) were combined to obtain the mediation (indirect) effect and a direct effect of the levels of the VGFA-R and problematic gaming while examining the direct effect of gender and IGDT-20. After a direct relationship between an independent (predictor) and dependent (outcome) variable was established, a mediation analysis was performed to determine if another variable explained a proportion of the relationship rather than presuming the entirety of the relationship was explained by one variable ${ }^{21}$. Each level of the VGFA-R was used as the primary predictor variable and IGDT-20 total score was used as the outcome variable, with gender 
and average hours played per typical week were used as mediating variables. All analyses were analyzed through SAS.

\section{Results}

Overall findings for the present study showed that the mediating variable(s) of gender and time spent gaming was significant in explaining the relationship between each function of the VGFA-R (attention, escape, sensory, tangible) and IGDT-20 total score (see Table 2 for results of mediation analysis).

\section{VGFA-R attention}

Gender. Findings showed a significant effect (coefficient $=-2.16 ; t=-2.10, p=.0366, \mathrm{CI}=[-$ $4.19,-0.14]$ ) of the independent variable VGFA attention score on the mediator gender (i.e., VGFA attention score was correlated with gender). VGFA attention had a significant effect with an estimate of $0.64(t=6.72, p<.0001, \mathrm{CI}=[0.45,0.83])$ but gender was not significant with an estimate of $1.17(t=.68, p=.495, \mathrm{CI}=[-2.20,4.55])$. Gender significantly mediated the relationship between VGFA attention and IGDT-20 total score. The direct effect of total IGDT20 with attention was significant $\left(\mathrm{R}^{2}=.13, \mathrm{~F}[2,301]=22.6, p<.0001\right)$.

Time spent gaming. Findings showed a significant effect (coefficient $=1.56 ; t=4.35$, $p<.0001, \mathrm{CI}=[0.86,2.27])$ of the independent variable VGFA attention score on the mediator hours spent (i.e., VGFA attention score was correlated with hours spent playing). VGFA attention had a significant effect with an estimate of $0.49(\mathrm{t}=5.33, p<.0001, \mathrm{CI}=[0.31,0.66])$, hours spent was significant with an estimate of $3.89(t=6.63, p<.0001, \mathrm{CI}=[2.73,5.04])$, and the relationship between VGFA attention and IGDT-20 total score. The direct effect of total IGDT20 with attention was significant $\left(\mathrm{R}^{2}=.24, \mathrm{~F}(2,301)=47.6, p<.0001\right)$.

\section{VGFA-R escape}


Gender. Findings showed a non-significant effect (coefficient=1.76; $t=1.86$, $p=.0636, \mathrm{CI}=[-0.10,3.63])$ with the independent variable VGFA escape score on the mediator gender, showing VGFA escape score was not correlated with gender. The following step in the mediation analysis established VGFA escape and gender effects on IGDT-20 total score. VGFA escape had a significant effect with an estimate of 1.37 $(t=17.45, p<.0001, \mathrm{CI}=[1.21,1.52])$ and gender was also significant with an estimate of $2.62(t=-2.02, p=.0442, \mathrm{CI}=[-5.17,-0.07])$. Gender significantly mediated the relationship between VGFA escape and IGDT-20 total score. The direct effect of total IGDT-20 with escape was significant $\left(\mathrm{R}^{2}=.50, \mathrm{~F}(2,301)=152.2, p<.0001\right)$.

Time spent gaming. Findings showed a significant effect (coefficient $=2.34 ; t=7.50$, $p<.0001, \mathrm{CI}=[1.73,2.96])$ of the independent variable VGFA escape score on the mediator hours spent (i.e., VGFA escape score was correlated with hours spent). The following step in the mediation analysis established VGFA escape and hours spent effects on IGDT-20 total score. VGFA escape had a significant effect with an estimate of $1.23(t=14.72, p<.0001, \mathrm{CI}=[1.07$, 1.39]) and hours spent was significant with an estimate of $1.77(t=3.57, p=.0004, \mathrm{CI}=[0.79$, 2.74]). The hours spent significantly mediated the relationship between VGFA escape and

IGDT-20 total score. The direct effect of total IGDT-20 with escape was significant $\left(\mathrm{R}^{2}=.52\right.$, $\mathrm{F}(2,301)=160.8, p<.0001)$.

\section{VGFA-R sensory}

Gender. Findings showed a non-significant effect $($ coefficient $=.97(t=1.08, p=.283, \mathrm{CI}=$ $[-0.80,2.73])$ of the independent variable VGFA sensory score on the mediator gender (i.e., VGFA sensory score was not correlated with gender). The following step in the mediation analysis established the VGFA sensory and gender effects on IGDT-20 total score. VGFA sensory had a 
significant effect with an estimate of $1.00(t=9.73, p<.0001, \mathrm{CI}=[0.79,1.20])$ and gender was not significant with an estimate of $-1.17(t=-0.73, p=.462, \mathrm{CI}=[-4.32,1.97])$. Gender significantly mediated the relationship between VGFA sensory and IGDT-20 total score. The direct effect of total IGDT-20 with sensory was significant $\left(\mathrm{R}^{2}=.24, \mathrm{~F}(2,301)=47.3, p<.0001\right)$.

Time spent gaming. Findings showed a significant effect (coefficient $=1.42 ; t=4.58$, $p<.0001, \mathrm{CI}=[0.81,2.03])$ of the independent variable VGFA sensory score on the mediator hours spent (i.e., VGFA sensory score was correlated with hours spent). The following step in the mediation analysis established the VGFA sensory and hours spent effects on IGDT-20 total score. VGFA sensory had a significant effect with an estimate of $0.83(t=8.37, p<.0001, \mathrm{CI}=[0.64,1.03])$ and hours spent was significant with estimate of $3.46(t=6.25, p<.0001, \mathrm{CI}=[2.37,4.55])$. The hours spent significantly mediated the relationship between VGFA sensory and IGDT-20 total score. The direct effect of total IGDT-20 with sensory was significant $\left(\mathrm{R}^{2}=.33, \mathrm{~F}(2,301)=72.6\right.$, $p<.0001)$.

\section{VGFA-R tangible}

Gender. Findings showed a significant effect (coefficient $=1.73 ; t=2.08, p=.0387, \mathrm{CI}=$ $[0.09,3.38])$ of the independent variable VGFA tangible score on the mediator gender (i.e., VGFA tangible score was correlated with gender). The following step in the mediation analysis established the VGFA tangible and gender effects on IGDT-20 total score. VGFA tangible had a significant effect with an estimate of $0.94(t=8.27, p<.0001, \mathrm{CI}=[0.72,1.16])$ and gender was not significant with an estimate of $-1.84(t=-1.11, p=.269, \mathrm{CI}=[-5.11,1.43])$. Gender significantly mediated the relationship between VGFA tangible and IGDT-20 total score. The direct effect of total IGDT-20 with tangible was significant $\left(\mathrm{R}^{2}=.19, \mathrm{~F}(2,301)=34.2, p<.0001\right)$. 
Time spent gaming. Findings showed a significant effect (coefficient=1.76; $t=6.23$, $p<.0001, \mathrm{CI}=[1.20,2.32])$ of the independent variable VGFA tangible score on the mediator hours spent (i.e., VGFA tangible score was correlated with hours spent). The following step in the mediation analysis established the VGFA tangible and hours spent effects on IGDT-20 total score. The VGFA tangible had a significant effect with an estimate of $0.71(t=6.18, p<.0001, \mathrm{CI}=[0.48$, $0.93])$ and hours spent was significant with an estimate of $3.41(t=5.72, p<.0001, \mathrm{CI}=[2.23,4.58])$. The hours spent significantly mediated the relationship between VGFA tangible and IGDT-20 total score. The direct effect of total IGDT-20 with tangible was significant $\left(\mathrm{R}^{2}=.26, \mathrm{~F}(2,301)=53.5\right.$, $p<.0001)$.

\section{Discussion}

The present study evaluated the differences between gender and hours playing videogames per week using the Video Game Functional Assessment-Revised (VGFA-R) and the 20-item Internet Gaming Disorder Test (IGDT-20) utilizing mediation analysis. The analysis demonstrated two predominant findings: (i) a significant gender difference between sub-factors of the VGFA-R, and (ii) total score the IGDT-20 related to the number of hours played per week between males and females. In both findings, the behavioral motivation of videogame play was critical in understanding the interplay between these variables. More specifically, the hours played per week across gender were complete mediations. This implies the severity of gaming play as defined by number of hours is a contextual variable within the pathology of addictive qualities of online gaming. The interaction between average hours played each week and gender significantly influenced the total score and each level of the IGDT-20.

One possible reason for this finding is that while number of hours of play is an important component in diagnosing an addiction to online gaming, gender was not a contributing factor. It 
must be noted that the amount of time spent gaming alone is not necessarily a good predictor of problem gamers and that many gamers play excessively with no problems ${ }^{22,23}$. Given the increased prevalence of females engaging with online gaming, further research needs to evaluate the impact of how the number of hours, gender, and the risk of developing IGD are associated. The VGFA-R sub-scores demonstrated meaningful gender differences in the present study. In particular, previous research ${ }^{19,20}$ has indicated the escape-maintained function of online gaming (i.e., avoiding school/job responsibilities) is significantly correlated with number of hours played weekly. However, in the present study a possible explanation for this finding is that gender does constitute a primary variable when evaluating an escape-maintained motivation of gaming and duration.

The present study has several limitations. Given that the study collected self-report data there is a possibility that data could have been biased due to social desirability bias and recall bias. The study was also cross-sectional, therefore, causality relating to any of the studied variables could not be determined. An attempt was made to mitigate some of these potential issues by installing smart technology within the Qualtrics survey to minimize potential biases, while providing a lottery-based incentive to individuals who completed the survey. While the data were collected via convenience sampling, it should be noted that in the present study the average mean age of individuals who engaged in online gaming (i.e., 29 years), and the increasing female usage of videogames matched the videogame industry's own findings ${ }^{6}$.

\section{References}


1. Mihara S, Higuchi S. Cross-sectional and longitudinal epidemiological studies of Internet gaming disorder: A systematic review of the literature. Psychiatry Clin Neurosci. 2017;71(7):425-444.

2. Feng W, Ramo DE, Chan SR, Bourgeois JA. Internet gaming disorder: Trends in prevalence 1998-2016. Addict Behav. 2017;75:17-24.

3. Griffiths MD, Kuss DJ, Billieux J, Pontes HM. The evolution of Internet addiction: A global perspective. Addict Behav. 2016;53:193-195.

4. Lopez-Fernandez O, Mannikko N, Kaariainen M, Griffiths MD, Kuss DJ. Mobile gaming and problematic smartphone use: A comparative study between Belgium and Finland. $J$ Behav Addict. 2018;7(1):88-99.

5. Lopez-Fernandez O, Williams AJ, Kuss DJ. Measuring female gaming: Gamer profile, predictors, prevalence, and characteristics from psychological and gender perspectives. Front Psychol. 2019;10:898.

6. Association ES. Esstential facts about the computer and video game industry 2018; http://www.theesa.com/article/essential-facts-computer-video-game-industry/.

7. Ciarrochi J, Parker P, Sahdra B, et al. The development of compulsive internet use and mental health: A four-year study of adolescence. Dev Psychol. 2016;52(2):272-283.

8. Su W, Kiraly O, Demetrovics Z, Potenza MN. Gender moderates the partial mediation of impulsivity in the relationship between psychiatric distress and problematic online gaming: Online survey. JMIR Ment Health. 2019;6(3):e10784.

9. (APA) APA. Diagnostic and statistical manual of mental disorders. 5th ed. Arlington, VA2013. 
10. Kuss DJ, Griffiths MD. Internet gaming addiction: A systematic review of empirical research. International Journal of Mental Health and Addiction. 2011;10(2):278-296.

11. Laconi S, Pirès S, Chabrol H. Internet gaming disorder, motives, game genres and psychopathology. Computers in Human Behavior. 2017;75:652-659.

12. Cotten SR, Jelenewicz SM. A disappearing digital divide among college students? Social Science Computer Review. 2016;24(4):497-506.

13. Dufour M, Brunelle N, Tremblay J, et al. Gender Difference in Internet Use and Internet Problems among Quebec High School Students. Can J Psychiatry. 2016;61(10):663-668.

14. Taylor TL. Play between Worlds: Exploring Online Game Culture. Cambridge, MA: The MIT Press. ; 2006.

15. Pontes HM, Kiraly O, Demetrovics Z, Griffiths MD. The conceptualisation and measurement of DSM-5 Internet Gaming Disorder: the development of the IGD-20 Test. PLoS One. 2014;9(10):e110137.

16. Pontes HM, Griffiths MD. Assessment of internet gaming disorder in clinical research: Past and present perspectives. Clinical Research and Regulatory Affairs. 2014;31(2-4):3548.

17. Baron RM, Kenny DA. The moderator-mediator variable distinction in social psychological research: conceptual, strategic, and statistical considerations. J Pers Soc Psychol. 1986;51(6):1173-1182.

18. Buono FD, Upton TD, Griffiths MD, Sprong ME, Bordieri J. Demonstrating the validity of the Video Game Functional Assessment-Revised (VGFA-R). Computers in Human Behavior. 2016;54:501-510. 
19. Sprong ME, Griffiths MD, Lloyd DP, Paul E, Buono FD. Comparison of the Video Game Functional Assessment-Revised (VGFA-R) and Internet Gaming Disorder Test (IGD-20). Front Psychol. 2019;10:310.

20. Buono FD, Griffiths MD, Sprong ME, Lloyd DP, Sullivan RM, Upton TD. Measures of behavioral function predict duration of video game play: Utilization of the Video Game Functional Assessment - Revised. J Behav Addict. 2017;6(4):572-578.

21. MacKinnon DP, Fairchild AJ, Fritz MS. Mediation analysis. Annu Rev Psychol. 2007;58:593-614.

22. Billieux J, Van der Linden M, Achab S, et al. Why do you play World of Warcraft? An indepth exploration of self-reported motivations to play online and in-game behaviours in the virtual world of Azeroth. Computers in Human Behavior. 2013;29(1):103-109.

23. Griffiths MD. The role of context in online gaming excess and addiction: Some case study evidence. International Journal of Mental Health and Addiction. 2009;8(1):119-125. 